\title{
Penser la fraternité pour éduquer le citoyen à la lumière de l'œuvre de Martha Nussbaum
}

\section{Stéphanie Péraud-Puigségur}

\section{(2) OpenEdition \\ Journals}

\section{Electronic version}

URL: http://journals.openedition.org/trema/5749

DOI: $10.4000 /$ trema.5749

ISSN: 2107-0997

\section{Publisher}

Faculté d'Éducation de l'université de Montpellier

\section{Electronic reference}

Stéphanie Péraud-Puigségur, «Penser la fraternité pour éduquer le citoyen à la lumière de l'œuvre de Martha Nussbaum », Tréma [Online], 53 | 2020, Online since 01 March 2020, connection on 10 December 2020. URL : http://journals.openedition.org/trema/5749; DOI : https://doi.org/10.4000/ trema. 5749

This text was automatically generated on 10 December 2020.

Trema 


\title{
Penser la fraternité pour éduquer le citoyen à la lumière de l'œuvre de Martha Nussbaum
}

\author{
Stéphanie Péraud-Puigségur
}

\section{Introduction}

1 L'Europe traverse un moment de fortes tensions politiques, où la rhétorique identitaire tourne à plein et où les médias nous alertent régulièrement sur les manifestations de xénophobie à l'égard de populations réfugiées qui essaient de fuir des conditions de vie rendues très difficiles par la guerre ou la misère. Dans le même temps, des associations ou de simples citoyens s'organisent pour les accueillir, pour partager un gite ou un moment de convivialité avec elles et témoignent de la richesse de ces rencontres humaines. De telles réactions montrent qu'au-delà de l'émoi suscité par les images de détresse des migrants en Méditerranée, des liens fraternels peuvent se tisser avec eux au quotidien dans les pays concernés. Dans ce contexte, comment l'éducation scolaire des jeunes générations peut-elle participer de cette dynamique fraternelle et prévenir plus largement les phénomènes de rejet qui fragilisent le lien social ? Pour contribuer à la réflexion sur cette question nous nous appuierons sur le travail mené par la philosophe américaine Martha Nussbaum sur les «émotions démocratiques " (Nussbaum, 2010/2011). Elle plaide en faveur d'une prise en compte de ces dernières pour contrebalancer la stigmatisation et la domination toujours menaçantes. Elle considère que même s'il semble impossible de «produire des gens capables de résister fermement à toute manipulation », on peut néanmoins, selon l'environnement culturel et le modèle éducatif privilégiés, « apprendre aux enfants à voir les nouveaux groupes migrants ou les étrangers comme une masse sans visage qui menace leur hégémonie [ou] au contraire leur apprendre à voir les membres de ces groupes comme des individus égaux, qui partagent des droits et des responsabilités communes » (Nussbaum, 2010/2011, p. 60). 
2 À titre liminaire, nous formulerons trois problèmes qui jalonnent la réflexion philosophique sur l'éducation à la fraternité avant de montrer, dans notre développement, en quoi la pensée de Martha Nussbaum permet de les approfondir et de les clarifier, sinon de les résoudre. Notre propos ne consistera donc pas à présenter des pratiques éducatives ou une étude empirique en contexte scolaire mais plutôt à déterminer philosophiquement le sens et les enjeux de certaines notions que peuvent mobiliser les acteurs de cette éducation à la fraternité : l'empathie, la sympathie, la compassion, l'émotion. L'analyse de l'approche conceptuelle de Martha Nussbaum et des principes pédagogiques qu'elle esquisse dans son ouvrage vise ainsi à alimenter la réflexion sur l'éducation à la fraternité en identifiant certaines difficultés principielles qu'elle soulève.

3 Le premier problème concerne la nature même de la fraternité qui peut varier selon le contexte familial, religieux, social ou politique auquel on se réfère. S'agit-il d'une valeur, d'un principe, d'un idéal, d'un devoir être ? Ou bien d'un sentiment, voire d'une émotion, d'une réalité qui s'éprouve et s'expérimente en situation? Il nous faudra resituer le propos de Martha Nussbaum par rapport à l'acception française du terme fraternité, héritée de l'approche républicaine qui la fonde. Nous aborderons ici la fraternité comme une émotion, elle-même articulée à un jugement, qui nous rend sensible au sort d'autrui et permet d'entretenir un lien direct entre les individus, pardelà leurs différences d'origine ou de condition. Martha Nussbaum n'utilise pas l'équivalent anglais du terme fraternité (Brotherhood) mais parle, selon les cas, d'empathie, de sympathie ou de « compassion rationnelle » pour évoquer ce qui peut unir les citoyens du monde en dépit de leur éloignement culturel ou social. Nous essaierons de comprendre les raisons de cette dernière formulation assez paradoxale qui vise à dépasser l'opposition caricaturale entre raison et émotion.

4 Le second problème porte sur les enjeux d'une éducation à la fraternité. Jusqu'à quel point l'école doit-elle et peut-elle s'en préoccuper? Pour Nussbaum, qui est libérale sur le plan politique, le risque d'intrusion dans la sphère privée ou de paternalisme institutionnel à l'égard des parents n'est jamais loin et il lui faut donc justifier cette intervention de l'école en matière d'éducation de la sensibilité, qui apparait au cœur de son projet éducatif. Nous examinerons pourquoi la formation de "citoyens du monde » est à ses yeux une mission centrale de l'école et une condition majeure de la vitalité des démocraties avant d'expliciter ce qui fait de la compassion rationnelle une émotion proprement démocratique. Même si elle reste très consciente des limites de celle-ci et ne l'envisage jamais sans des lois et institutions justes à même d'assurer une certaine stabilité politique, elle développe une argumentation serrée pour justifier la place essentielle qu'elle lui accorde dans son projet humaniste. En France, le contexte scolaire et universitaire apparaît par certains aspects bien différents de ce dont parle Nussbaum à propos du système américain. En effet, les programmes actuels évoquent le triptyque républicain comme un élément incontournable du parcours de l'élève et la fraternité comme une valeur essentielle de l'institution. Mais à examiner les pratiques scolaires ou certains propos de professeurs, il faut bien admettre qu'elle ne semble guère aisée à enseigner ou à évaluer ${ }^{1}$. En dépit de ces difficultés, les attentats survenus en France à partir de 2015 et les programmes d'Enseignement Moral et Civique mis en œuvre la même année ont relancé le questionnement sur les enjeux de cette éducation à la fraternité. Dès lors, peut-on se contenter de discours favorables à une éducation à la fraternité dans une école Républicaine mettant au cœur de son projet la formation 
des citoyens sans donner aux acteurs éducatifs des clés pour agir efficacement auprès des élèves?

5 Ceci nous conduit au dernier problème que nous aborderons, celui des moyens de réalisation de cette éducation à la fraternité et de la formation de la sensibilité qu'elle requiert. La porte est étroite entre un discours purement injonctif adressé aux enseignants comme aux élèves et des dérives sentimentalistes contestables, dans un contexte où l'usage idéologique des affects tend parfois à infiltrer le champ scolaire. En s'appuyant sur une position équilibrée, Martha Nussbaum propose des principes d'action aux enseignants afin de donner corps à cette éducation de la sensibilité et de la pensée critique, qu'elle appelle sans cesse de ses vœux. En mettant en évidence les liens entre sa propre pensée et celle de grands pédagogues Européens, Américains et Indiens, elle nous donne ainsi indirectement des pistes pour développer chez les jeunes la "compassion rationnelle», cette émotion démocratique appuyée sur l'imagination narrative et la capacité de se mettre à la place d'autrui. Le détour par ces réflexions et expérimentations parfois oubliées constitue également un appel à la cohérence entre discours et actes en matière d'éducation aux valeurs.

\section{La fraternité : une notion complexe éclairée d'un jour nouveau par Martha Nussbaum}

\section{Qu'est-ce que la fraternité ?}

6 L'évolution de la notion analysée par les historiens et les philosophes permet de comprendre sa surdétermination sémantique. D'abord référée au lien familial puis, par extension, au lien entre croyants issus d'un même "père » divin, la notion s'est peu à peu laïcisée et signifie aujourd'hui plus largement le lien entre les membres d'un même corps politique, raison pour laquelle elle figure dans notre devise ${ }^{2}$. Il faut rappeler la place de la notion dans la Révolution Française, où, comme le dit Mona Ozouf, elle oscille entre la «fraternité euphorique » de la fête de la Fédération célébrée le 14 juillet 1790, où les barrières sociales semblent abaissées et la « fraternité-verrouillée » (Furet \&. Ozouf) manifeste dans l'ambivalence de la devise «la fraternité ou la mort». Elle n'est cependant inscrite dans la devise républicaine qu'en 1848, en signe de protestation contre une humanité divisée en classes ou en "races » et apparaît alors comme un contre-feu essentiel au pur calcul d'intérêt encouragé par une lecture individualiste du contrat social. Par ailleurs, la notion oscille sans cesse entre un niveau infra-étatique, comme sentiment préparant le lien politique ${ }^{3}$ et un niveau supraétatique, comme dépassement des logiques patriotiques lors de combats politiques ou sociaux transnationaux. Pensons à la fraternisation entre soldats ou entre ouvriers d'origines différentes qui témoignerait d'un lien universel transcendant les frontières et les affrontements politiques suscités par l'histoire. Quoi qu'il en soit, c'est souvent dans des circonstances exceptionnelles mettant à l'épreuve le lien humain que la fraternité est invoquée, de façon soudaine et parfois très transgressive par rapport à l'ordre social et politique en place, comme le montrent les exemples de la révolution de 1848 déjà évoqué et d'autres grands moments de fraternité républicaine comme les grèves de 1936 ou les évènements de mai 1968 et, plus récemment, les manifestions de Janvier 2015 suite aux attentats de Paris. On peut néanmoins se demander s'il est 
possible de fonder sur les affects qui s'y expriment un lien social solide et une action pérenne à même de changer la vie des concitoyens.

7 Un second élément participant de la complexité de la notion tient à son caractère ouvert ou fermé selon les cas. Qui sont les frères: les membres d'une famille, d'une communauté religieuse, d'un parti, d'un État? Ou potentiellement tous les hommes dès lors qu'ils se reconnaissent dans un projet partagé ou une expérience commune de la vulnérabilité, pour reprendre un terme cher à Nussbaum ? Le resserrement des liens au sein d'un collectif fraternel peut en effet parfaitement s'assortir d'une indifférence voire d'une haine assumée à l'égard de l'autre, dès lors qu'il ne pense pas et ne croit pas la même chose, ou présente des caractéristiques physiques ou culturelles différentes de celles des membres de ce groupe. Ce «biais de proximité » (Hoffman, 2000) génère un risque de repli de la fraternité sur un collectif restreint ou sur une élite et oblige à une vigilance de chaque instant pour résister aux logiques de rejet de l'autre.

8 Enfin la nature même de la fraternité pose question : la fraternité est-elle une valeur, un idéal, un principe politique ou un affect? Ce problème est relativement inextricable et selon que l'approche est philosophique, historique ou juridique, le même terme peut avoir différentes significations et valeurs. Or, le statut qu'on accorde à la fraternité en détermine l'usage qui peut lui-même rétroagir sur le sens de la notion en fonction des différents contextes dans lesquels elle se déploie. Ainsi, un principe permet une déclinaison politique ou législative sous forme de droits. On peut néanmoins remarquer que le principe de solidarité a longtemps été préféré par les juristes à celui de fraternité même si des décisions récentes semblent changer la donne ${ }^{4}$. Un idéal appelle, quant à lui, la réalisation d'un projet de société alternatif. Pensons, de ce point de vue, à l'idée d'une fraternité laïcisée et universelle appuyée sur la reconnaissance d'autrui comme frère humain, qui irrigue aussi bien la franc maçonnerie que la pensée républicaine de l'école publique ou les pédagogies nouvelles. Si, enfin, on envisage la fraternité comme émotion ou sentiment ${ }^{5}$, se pose alors la question de son origine, de ses effets et des moyens de la favoriser ou de l'entretenir ou a contrario, des obstacles à son plein épanouissement. Nous privilégierons ici cette dernière acception de la fraternité conçue comme affect dont Martha Nussbaum nous permet d'éclairer la compréhension dans le cadre de la réflexion qu'elle mène sur la formation des citoyens aujourd'hui.

\section{Penser la fraternité à la lumière de la théorie des émotions démocratiques de Nussbaum}

9 Cette philosophe américaine a publié en 2010 un ouvrage manifeste analysant deux modèles d'éducation en concurrence aujourd'hui : l'un qu'elle considère comme tourné vers le profit et qui menacerait à terme la paix civile et mondiale, l'autre centré sur la formation de citoyens capables de maintenir le lien démocratique vivace et dans lequel les humanités joueraient un rôle essentiel. Ce texte, suggestivement intitulé Not For Profit, Why Democracy Needs The Humanities a été traduit en français par Solange Chavel en 2011 sous le titre Les émotions démocratiques. Cette désignation met l'accent sur l'aspect le plus original de cet ouvrage qui donne une place essentielle à la réflexion sur la place des émotions dans la formation des jeunes générations. Nussbaum s'inscrit ainsi dans la continuité de penseurs antiques (Platon, Aristote, les stoïciens) et modernes (Spinoza, Rousseau, Mill) en s'interrogeant sur l'impact des passions ou affects dans le champ politique en général et dans l'éducation des citoyens en 
particulier. Cet ouvrage nous semble ainsi particulièrement fécond pour éclairer certains des débats les plus actuels sur la question de l'éducation à la fraternité et sur le rôle qu'y joue la sollicitation de la sensibilité des élèves.

La philosophe se saisit de cette question du lien fraternel dans un vocabulaire qui lui est propre mais qui renvoie en réalité à des concepts hérités des philosophies morales antiques et modernes. Elle les revisite dans le contexte contemporain qu'elle identifie comme une remise en cause des principes d'une éducation démocratique et humaniste à l'échelle mondiale. Pour comprendre la part essentielle qu'elle accorde aux émotions démocratiques, il faut saisir la façon singulière dont Nussbaum envisage l'émotion. Sa théorie des émotions morales et politiques s'appuie sur une lecture critique des stoïciens. En même temps qu'elle leur emprunte l'idée que toute émotion implique un jugement de valeur, Nussbaum refuse d'ériger en idéal le sage stoïcien indépendant à l'égard de ses pairs ou de la Fortune. La compréhension de notre dépendance à l'égard des circonstances ou d'autrui doit nous amener à reconnaitre notre vulnérabilité, laquelle est mise en scène de façon exemplaire dans la tragédie. Tout déni de cette fragilité humaine ne peut d'après elle que nous égarer moralement et politiquement. Elle inverse donc la perspective stoïcienne en insistant sur notre dépendance à l'égard des autres, que notre situation d'autonomie momentanée et apparente peut nous conduire à oublier, mais que la vieillesse ne manquera pas de nous rappeler.

Elle insiste par ailleurs sur le fait que les émotions sont « des réactions intelligentes à la perception de la valeur » (Nussbaum, 2001, p. 1 ; Goldstein, 2011, p. 36). À ce titre, elles appellent une explicitation et au-delà, une discussion sur ce qui vaut vraiment à nos yeux. Il faut en quelque sorte les déplier pour saisir la façon dont le sujet qui les ressent hiérarchise les valeurs et ainsi révéler les jugements moraux ou politiques impliqués dans l'affect en question. Celui-ci peut être la colère, l'admiration, la compassion, l'amour, le mépris, le dégoût ou la haine, ce que les auteurs classiques, Spinoza ou Descartes par exemple, appellent les "passions». C'est d'ailleurs ce genre d'analyse qu'elle va s'appliquer à conduire à partir d'exemples littéraires dans nombre de ses ouvrages, en particulier ceux offerts par les romans et nouvelles d'Henry James, où les émotions des personnages sont passées au crible pour identifier les positions de principe qui sont les leurs et aiguiser l'imagination morale de ses étudiants et de ses lecteurs.

Examinons le cas de la compassion, auquel Nussbaum accorde une importance particulière parmi les émotions démocratiques dans son ouvrage. Celle-ci concentre en effet quelques-unes des questions les plus intéressantes sur cette place de l'émotion dans l'éducation du citoyen. Pour éviter toute confusion, il nous semble d'abord important de distinguer l'empathie (empathy) d'une part et la sympathie (sympathy), la compassion (compassion) ou la pitié (pity) d'autre part, ces trois derniers vocables étant fréquemment associés dans ses textes. En effet, chacun de ces termes renvoie à une capacité de se décentrer afin de se mettre à la place d'autrui et d'accéder à une compréhension de ses états affectifs ou mentaux. Mais l'empathie n'exige pas pour autant une impulsion à agir en vue d'améliorer le bien-être de la personne considérée alors que c'est le cas de la sympathie, de la compassion ou de la pitié. Ces formulations assez simples en apparence suscitent pourtant bien des questions: que signifie exactement «se mettre à la place d'autrui »? Est-ce réellement possible ou s'agit-il d'une façon imagée de se représenter ce qui se joue à cette occasion? Qu'est-ce qui nous permettrait en réalité d'opérer un tel décentrement? Quel est au juste le rôle de 
l'imagination dans ce processus largement exploré par Rousseau dans l'Emile, par exemple, lorsqu'il s'interroge sur la nature de la pitié ? Et surtout, point central pour aborder notre question de la fraternité, comment s'opère le passage de l'empathie à la sympathie? Comme le remarque justement Nussbaum, l'empathie peut en effet être une arme redoutable dans les mains d'un bourreau sadique qui pourrait par ce biais tourmenter sa victime sans jamais ressentir de sympathie pour elle. Si elle évoque à plusieurs reprises les mécanismes de l'empathie dans son ouvrage, Nussbaum s'intéresse avant tout à la sympathie ou à ce qu'elle appelle plus fréquemment encore la compassion. Les deux termes signifient d'ailleurs étymologiquement la même chose, même si la compassion peut sembler une émotion plus intense et plus directement attachée à la souffrance d'autrui. On peut en effet ressentir de la sympathie pour quelqu'un qui ne souffre pas, mais pas de la compassion. Elle définit cette dernière comme une "émotion douloureuse occasionnée par la conscience de l'infortune imméritée d'une autre personne" (Nussbaum, 2001, p. 306) en s'inspirant de la définition d'Aristote ${ }^{6}$.

13 Ajoutons immédiatement que Nussbaum se garde de sacraliser l'émotion et de la considérer comme une boussole infaillible pour l'action et le jugement. La compassion, comme toutes les émotions n'est pas absolument fiable. Comme il y a d'injustes colères, il y a des compassions fautives, fondées sur la reconnaissance excessive d'un tort mineur fait à autrui. La compassion prend tout son sens lorsqu'elle s'exerce à l'égard d'une personne ne bénéficiant pas de ressources minimales pour vivre dignement. Nussbaum fait d'ailleurs la liste de ces biens de base dont le manque devrait susciter la compassion, dans son ouvrage sur les capabilités : «la vie, les personnes aimées, la liberté, la nourriture, la mobilité, l'intégrité physique, la citoyenneté, l'abri» (Nussbaum, 2011). La compassion ne suffit pas, il faut nécessairement y ajouter une dimension normative pour éviter de tomber dans une sensiblerie aveugle à la réalité du mal subi par autrui. C'est pourquoi elle parle de « compassion rationnelle " (Nussbaum, 2001, p. 425) et de la nécessité d'assortir la compassion d'une réflexion approfondie sur la valeur des biens de base et sur les personnes qui doivent susciter notre attention, qu'elles soient proches ou éloignées de nous. Point de vue qu'elle résume ainsi : « [...] ce que nous voulons cultiver, c'est la compassion appropriée fondée sur un jugement raisonnable » (Nussbaum, 2001, p. 447). Il s'agit donc de construire une théorie éthique correcte, fondée sur une juste estimation des biens essentiels et des conditions d'un accès effectif à ces biens, ce qu'elle nomme "capabilités ", pour que l'émotion ne soit pas dévoyée et puisse être mise au service de la fraternité. Malgré ses limites et les réserves qu'elle suscite, la compassion apparait comme l'une des pierres angulaires de l'éducation démocratique que Nussbaum appelle de ses vœux.

\section{Les enjeux d'une éducation à la « compassion rationnelle »}

\section{Accompagner l'enfant dans son développement rationnel et émotionnel}

14 Le projet éducatif de Nussbaum s'appuie sur deux piliers complémentaires. Pour éduquer le citoyen, elle insiste en permanence sur la nécessité d'une pédagogie socratique visant à développer la rationalité et l'esprit critique de l'élève. Ce point de 
vue n'est guère original même si elle constate que l'évolution des systèmes éducatifs à l'échelle mondiale se concentre sur la formation de travailleurs productifs et hyperspécialisés plutôt que sur celle de citoyens critiques sensibilisés à la chose publique et au cosmopolitisme. Ce qui l'est davantage est l'accent qu'elle met en permanence sur l'importance d'une éducation des émotions de ces mêmes élèves en vue de soutenir le projet démocratique. Nussbaum s'interroge en effet sur les freins à la fraternité, sur les mécanismes psychologiques et sociaux qui entretiennent des identités concurrentes voire inconciliables au niveau national ou international, donnant ainsi naissance à l'idéologie mortifère du "choc des civilisations ", laquelle entretient à son tour des émotions propices au rejet et à la violence. Pour lutter contre ces dérives, il faut apprendre aux citoyens à lutter en eux-mêmes comme chez les autres contre les émotions qui, faisant obstacle au lien fraternel, minent la démocratie : «[...] la lutte politique pour la liberté et l'égalité doit d'abord être une lutte à l'intérieur de chaque personne, tandis que la compassion et le respect luttent avec la peur, l'avidité et l'agressivité narcissiques" (Nussbaum, 2010/2011, p.41). Lorsque le discours rationnel, politique, moral ou juridique reste inopérant, lorsque les affects de haine ou de mépris l'emportent sur le jugement, il faut faire jouer passion contre passion, selon une stratégie assez similaire à celle envisagée par Descartes dans Le traité des passions ${ }^{7}$.

Pour asseoir sa démonstration et préciser les enjeux d'un tel travail sur l'émotion compassionnelle, elle s'appuie sur sa lecture de Rousseau comme sur celle de psychologues et sociologues ayant étudié la place des émotions dans la formation et l'action des sujets. Nussbaum rend ainsi hommage au philosophe genevois dans la mesure où il pense une éducation capable de modeler les sentiments moraux. En évoquant l'Émile, elle reconstitue en quelque sorte le parcours émotionnel du jeune enfant et ses issues contradictoires en fonction de l'éducation qu'il reçoit. "Ce parcours idéal -narcissisme, vulnérabilité, honte, dégoût et compassion- me semble être le cœur d'une éducation tournée vers la citoyenneté démocratique " (Nussbaum, 2010/2011, p.55). La prise de conscience par le jeune enfant de sa vulnérabilité et de sa dépendance à l'égard des adultes génère un sentiment de honte associé au dégoût provoqué par les marques de son animalité que la culture l'amène à rejeter. L'identification de cette projection de la honte et du dégoût sur autrui permet d'expliquer les mécanismes de l'indifférence, du rejet et du racisme, de l'obsession de la pureté et, peut-être, de les prévenir. Les deux voies ouvertes par Rousseau pour éviter le retournement de la détresse originelle en dégoût à l'égard de toutes les formes de vulnérabilité perçues comme des faiblesses sont le développement précoce de l'autonomie pratique de l'enfance et la pitié. Celle-ci l'amène non seulement à envisager la situation et le ressenti de l'autre (empathie), mais à agir in fine en sa faveur (passage à la sympathie ou à la compassion). L'éducation et, plus globalement, la culture doivent donc permettre de dépasser cette tendance universelle au rejet de l'autre et de développer et d'entretenir ce lien fraternel.

\section{Lutter contre l'indifférence}

16 Nussbaum s'appuie sur des travaux de psychologie sociale ${ }^{8}$ pour comprendre les mécanismes d'activation ou de désactivation de l'empathie et de la sympathie à l'égard de personnes différentes de soi ou extérieures au groupe social d'appartenance. Elle montre bien le caractère réversible de la compassion puisque l'intensification de cette 
émotion à l'égard des membres du groupe de pairs peut s'assortir d'une totale indifférence à la souffrance de ceux qui sont perçus comme ennemis ou infra humains. Elle insiste sur l'importance du contexte pour orienter ces émotions. La déresponsabilisation des sujets, l'absence d'une voix critique à l'intérieur du groupe de pairs et l'enrôlement idéologique conjugués conduisent ainsi certains sujets éduqués et habituellement sensibles à la souffrance humaine à commettre des actes humiliants ou criminels à l'égard d'autres personnes, comme en témoignent notamment les travaux de Zimbardo (2007), reprenant les constatations faites dès 1972 à partir de l'expérience de la prison de Stanford sur les effets de la situation carcérale. En inversant la perspective, on comprend mieux ce que serait une éducation véritablement démocratique et fraternelle. Elle s'efforcerait de renforcer le sentiment de responsabilité personnelle des sujets, de développer leur capacité à élever une voix critique lorsqu'ils sont en minorité et de les amener à voir les personnes différentes comme des individus distincts et dignes de respect plutôt que comme une masse indifférenciée et anonyme. C'est à ce dernier niveau que va jouer le plus efficacement l'éducation à la compassion, appuyée sur l'imagination de l'enfant. Ce programme éducatif ambitieux reste encore relativement général, prescriptif et peu opératoire. Nussbaum se tourne donc vers certains penseurs de l'éducation dont les principes et les expériences pédagogiques diverses nous aident à imaginer ce que pourrait être cette éducation à la fraternité et à nous orienter encore aujourd'hui.

\section{Comment éduquer à la « compassion rationnelle »?}

\section{La voie étroite de l'éducation à la fraternité}

Même si nous reconnaissons avec Nussbaum le caractère essentiel de la compassion pour éduquer à la fraternité, la question des moyens à mobiliser pour réaliser cette éducation reste entière. En effet, la compassion ne se décrète pas mais se cultive et s'entretient. Dès lors, comment apprendre aux jeunes générations à faire un usage éclairé de cette émotion? En ce domaine, le risque d'une imposition artificielle, d'une dérive sentimentaliste, voire d'une instrumentalisation politique de la fraternité n'est jamais loin, comme le montrent les débats des révolutionnaires français sur l'éducation morale et civique à dispenser aux futurs citoyens. Les projets éducatifs concurrents de Condorcet et Lepeletier de Saint-Fargeau ${ }^{9}$ soulèvent clairement le problème de la place à accorder au sentiment fraternel dans l'éducation révolutionnaire. Les dérives affectives présentes dans le modèle d'éducation spartiate de Lepeletier de SaintFargeau, immédiatement dénoncées par Condorcet qui privilégie l'instruction comme voie d'accès à un savoir rationnel peuvent sembler très loin de nous. Cependant, les tentatives d'un ancien Président de la République française d'introduire des parrainages d'enfants déportés à Auschwitz par des élèves de dix ans ou d'exalter le sentiment fraternel par la lecture émouvante de la lettre de Guy Moquet ont suscité un certain émoi et de légitimes questionnements dans le corps enseignant comme dans le grand public ${ }^{10}$. Ces dispositifs invitant de jeunes élèves à s'identifier au destin tragique d'un condamné ou d'un déporté furent en effet perçus comme des errements de la "politique compassionnelle» critiqués sévèrement par Myriam Revault d'Allonnes (2008). Ces écueils redoutables tout à fait contraires à la nécessité de former la raison et l'esprit critique des jeunes générations dans l'héritage condorcétien qui est le nôtre ne doivent cependant pas nous conduire à renoncer à éduquer à la fraternité. Nussbaum 
nous aide en effet à comprendre à quelles conditions certaines démarches pédagogiques permettent de développer efficacement les émotions démocratiques.

\section{Le jeu et la littérature pour éduquer à la fraternité}

18 S'appuyant sur son expérience d'enseignement à de futurs juges à l'université de Chicago, la philosophe affirme la nécessité d'apprendre aux jeunes à se mettre à la place d'autrui ${ }^{11}$. Contre un discours simplificateur valorisant la mise à distance de l'émotion dans l'évaluation des situations qu'ils auraient à juger, au nom de l'impartialité, elle insiste au contraire sur l'importance de pouvoir se mettre à la place des différents protagonistes pour comprendre la complexité d'une situation et juger de façon équitable. Elle développe une conception élargie de la raison, héritée du concept aristotélicien de phronésis, terme habituellement traduit par "prudence». Celle-ci permet l'évaluation de situations particulières grâce aux ressources de l'imagination morale. Cette disposition s'acquiert et suppose un exercice répété qui, au-delà du cas isolé des juges, devrait être proposé à chaque élève dans sa formation afin de démultiplier ses points de vue sur le monde et de l'amener à prendre du recul sur sa propre façon de hiérarchiser les valeurs à travers l'analyse collective de situations précises. Ce travail régulier permettrait d'élargir sa compréhension grâce à l'imagination et de développer sa capacité de juger grâce aux discussions menées à partir d'expériences et d'émotions partagées. Quel type de situation proposer aux élèves et aux étudiants pour $\mathrm{y}$ parvenir? Nussbaum suggère de prendre systématiquement appui sur la littérature et le jeu pour apprendre aux élèves «à se mettre à la place d'autrui ».

Elle accorde une place essentielle à la littérature et extrapole à partir du travail qu'elle a pu mener avec ses étudiants et dans ses écrits sur différentes œuvres littéraires, notamment celles d'Henry James qu'elle affectionne particulièrement du fait des situations de dilemmes moraux dans lesquelles se trouvent pris ses héros. Cette promotion de la littérature comme instrument de formation s'appuie sur la conception de la rationalité évoquée plus haut. Cette sagesse pratique à laquelle il faut former les élèves « ne peut pas être une science systématique qui ne s'occuperait que de principes universels et généraux » et exige qu'on accorde une "priorité [aux] jugements concrets et situés, d'une sorte plus informelle et intuitive que n'importe quel système " (Nussbaum, 1990/2010, p. 106). Comme le dit encore Chavel, cette approche exige de dépasser la classique opposition entre sensibilité et raison ${ }^{12}$ puisque «nos efforts de théorisation morale et nos efforts de jugement n'ont de sens que comme explicitation d'une réalité singulière qui est d'abord éprouvée, vue, sentie » (2011, p. 54).

Inversement, il ne s'agit pas seulement, à travers les exemples présents dans les textes littéraires, de faire ressentir l'injustice, ou de rendre le lecteur compatissant à l'égard du personnage. Cette sensibilité morale appelle et exige un véritable travail rationnel et théorique de ce matériau émotionnel par les élèves. Il ne suffit pas de ressentir soimême des émotions, il faut aussi comprendre ce que pensent ou ressentent les personnages et expliciter les raisonnements ou valeurs qui les sous-tendent. « La thèse de Nussbaum, qu'elle évoque avec beaucoup de nuances, est que certaines émotions, dans certaines conditions, sont également notre moyen de reconnaître et d'accéder aux valeurs morales» (Chavel, 2011, p. 48). À titre d'illustration, pour compléter la connaissance théorique ou juridique de ce qu'est la discrimination, Nussbaum invite les 
éducateurs à s'appuyer sur la littérature pour amener les élèves à expérimenter d'une certaine façon ce que cela fait d'être discriminé. Pour le dire autrement, on peut tout à fait apprendre aux élèves à identifier ce que sont les discriminations, rappeler les lois qui ont cours à ce sujet, mais cela ne suffit pas encore. Il faut que l'élève puisse, en quelque sorte, se mettre à la place de la personne discriminée ou sentir à quoi ressemble le monde de celui qui discrimine, pour qu'il change son comportement ${ }^{13}$. La littérature est, du fait de sa forme originale, un démultiplicateur d'expériences et de points de vue sur la réalité. Elle complexifie la compréhension que nous en avons, en même temps qu'elle l'affine et nourrit notre réflexion politique et morale. Ceci est d'autant plus vrai que s'y ajoute la pratique du débat interprétatif ou de la discussion à visée philosophique appuyée sur des textes ouverts, non univoques. Elle permet aux élèves de dépasser leurs premières impressions et de construire une analyse collective et argumentée de l'expérience ainsi partagée à partir des œuvres, tout en affinant progressivement l'intelligence des situations de discrimination, de rejet, de domination. Nussbaum ne se fait cependant aucune illusion sur le fait que l'expérience littéraire, même discutée et analysée pourrait à elle seule permettre la formation morale du jugement et l'accès à une fraternité authentique : le passage de l'empathie à la sympathie n'est jamais garanti et les pires bourreaux peuvent être en même temps des lettrés raffinés, comme l'Histoire nous l'a montré.

Mais la faiblesse de l'imagination morale et la pauvreté de l'expérience sensible de situations engageant des valeurs morales et/ou politiques sont certainement des obstacles à la compassion. Se priver de cette ressource serait d'autant plus regrettable d'après nous que la littérature de jeunesse offre aujourd'hui de très nombreuses occasions pour les élèves d'explorer le rapport à soi-même et aux autres, grâce au " paravent du personnage » (Chirouter, 2007) et à la qualité proprement littéraire des textes. La littérature permet ainsi de renforcer, au-delà des capacités de raisonnement, les ressources émotionnelles et imaginatives des élèves pour les aider à se comprendre eux-mêmes et à comprendre les autres, condition nécessaire bien que non suffisante d'un véritable sentiment fraternel.

Le jeu est une autre piste à suivre pour décliner pédagogiquement ces réflexions de Nussbaum. Entendons-le aux différents sens du terme, même si elle privilégie le jeu de rôle et le "jeu " théâtral pour introduire une sorte de "jeu» dans le "je ", afin de susciter un recul réflexif sur nos habitudes, nos émotions et nos pensées. S'appuyant sur Winnicott, Nussbaum (2010/2011, p. 126) considère que le jeu est par ailleurs un outil essentiel pour apprendre à négocier la relation avec les pairs et faire l'expérience de l'altérité d'une façon non menaçante car plus indirecte qu'habituellement. Elle rappelle d'ailleurs que cette pratique a été développée par le grand poète et penseur indien Rabindranath Tagore qui, dans son école de Santiniketan, « utilisait les jeux de rôles tout au long de la journée d'école, les positions intellectuelles étant examinées en demandant aux enfants de prendre des postures de pensée qui ne leur étaient pas familières " (Ibid., p.132). L'enjeu de formation de l'esprit à la critique et à l'argumentation était évidemment présent dans cette pratique, mais Tagore recherchait aussi une manière de cultiver la sympathie. Il utilisait également ce procédé pour explorer la question religieuse, sensible du fait des tensions entre hindous et musulmans en Inde dès cette époque, en demandant à ses élèves de " jouer " des cultes religieux inconnus pour «comprendre l'étranger grâce à la participation imaginative » (Ibid.). Tagore privilégiait également le jeu théâtral et la danse pour offrir à ses élèves une réelle ouverture d'esprit via le travail sur les rôles et les postures 
associées, notamment pour libérer les filles de l'intériorisation de normes corporelles aliénantes et les garçons d'une posture de pseudo-virilité excluant toute culture de la sensibilité. Ces expériences déjà anciennes trouvent cependant des échos dans des démarches aujourd'hui mobilisées dans le cadre scolaire. Pensons au théâtre-forum développé par Augusto Boal au Brésil et en France mais aussi au «jeu des trois figures", conçu par Serge Tisseron pour prévenir le harcèlement scolaire dès la maternelle. L'objectif de ces jeux de rôle scénarisés par les élèves est de leur éviter de s'enfermer précocement dans des identifications figées à l'agresseur, à l'agressé ou au "sauveur " et de trouver collectivement des ressources pour élever une voix distincte lorsqu'une injustice survient ou qu'une situation d'oppression se fait jour.

\section{Conclusion}

Nous avons rendu compte du travail conceptuel de Martha Nussbaum sur les émotions démocratiques parce qu'il nous semble éclairant pour les éducateurs comme pour les chercheurs et permet de mieux comprendre les enjeux de l'éducation sensible des jeunes générations. L'intérêt des diverses pratiques éducatives qu'elle évoque est d'amener les élèves à vivre imaginairement des positions de vulnérabilité ou de stigmatisation et à développer des capabilités pour échapper aux stéréotypes et à l'indifférence. La littérature et les arts, par leur forme même, nous mettent ainsi en position d'expérimenter des émotions, des positions sociales et des dilemmes propices au développement de notre imagination morale et de la compassion rationnelle. La découverte de cette philosophie de l'éducation originale, nourrie de références antiques tout en étant ancrée dans les questionnements politiques et sociaux les plus contemporains ouvre ainsi de nouvelles perspectives de recherche. Elle nous incite à poursuivre collectivement nos travaux pour vérifier la pertinence d'une telle analyse en contexte scolaire et à construire des outils pour mesurer de façon rigoureuse les progrès de cette compassion rationnelle chez les élèves. Néanmoins, même si son intérêt se trouvait confirmé, cette éducation scolaire aux " émotions démocratiques " ne suffirait pas encore pour prévenir le racisme ou l'insensibilité à l'égard d'autrui, ce que Nussbaum ne cesse de répéter tout en nous aidant à mieux comprendre l'articulation féconde de la rationalité et de la sensibilité qu'exige la formation du citoyen. C'est le travail conjoint de la famille et de l'école, appuyé sur la formation de l'esprit critique et l'apprentissage du droit, qui permettrait, en complément de cette expérience sensible, une véritable éducation à la fraternité. La discussion rationnelle, à visée interprétative ou philosophique, peut aider à faire expliciter les valeurs en jeu dans le «matériau émotionnel» livré par les élèves et à comprendre leur hiérarchisation selon les points de vue expérimentés, en référence à des normes partageables et discutables. Quant au droit, il ne se contente pas de refléter les normes engagées dans nos émotions, mais il les fait aussi évoluer comme le montre l'exemple du recul de la tolérance à l'égard de certains comportements comme le harcèlement sexuel ou scolaire. Ajoutons qu'un système scolaire qui déploierait de telles pratiques éducatives visant à susciter des "émotions démocratiques» sans modifier structurellement son propre fonctionnement ségrégatif et inégalitaire ne pourrait que se heurter à un échec ou à l'accusation méritée d'incohérence. Comment l'école peutelle encourager la fraternité dans les programmes de façon crédible sans lutter efficacement contre la reproduction sociale qu'elle génère par son fonctionnement même? Si l'éducation à la fraternité n'est pas conduite en même temps qu'une 
réflexion globale sur l'institution scolaire et ses mécanismes d'exclusion et de stigmatisation, elle ne peut avoir qu'un effet cosmétique. Il n'y a pas d'éducation à la fraternité possible dans une école qui ne serait pas elle-même réellement fraternelle.

\section{BIBLIOGRAPHY}

Baczko, B. (éd). (1982). Une éducation pour la démocratie, textes et projets de l'époque révolutionnaire. Paris : Garnier frères.

Chalier, C. (2003). La fraternité, un espoir en clair obscur. Paris : Buchet-Chastel.

Chavel, S. (2011). Se mettre à la place d'autrui, l'imagination morale. Rennes : P.U.R.

Chirouter, E. (2007). Lire, réfléchir et débattre à l'école élémentaire. La littérature de jeunesse pour aborder des questions philosophiques. Paris : Hachette.

Clark, C. (1997). Misery and Company. Sympathy in Everyday Life. Chicago: University of Chicago Press.

Descartes, R. (1996). CEuvres complètes. Paris : Vrin.

Goldstein, P. (2011).Vulnérabilité et autonomie dans la pensée de Martha C. Nussbaum. Paris : PUF.

Hoffman, M. (2000). Empathy and moral development : Implications for Caring and Justice. New York: Cambridge University Press, (traduction française : (2008) Empathie et développement moral. Les émotions morales et la justice. Grenoble : PUG).

Nussbaum, M. (1990). Love's Knowledge - Essays on Philosophy and Littérature. Oxford: Oxford University Press, 1990, traduction française par Chavel, S. (2010). La connaissance de l'amour : Essais sur la philosophie et la littérature. Paris : Cerf.

Nussbaum, M. (1995). Poetic Justice, The Literary Imagination and Public Life. Boston : Beacon Press, traduction française par Chavel S. (2015). L'art d'être juste. Paris : Flammarion.

Nussbaum, M. (2001). Upheavals of Thought: the Intelligence of Emotions. Cambridge: Cambridge University Press.

Nussbaum, M. (2006). Frontiers of Justice: disability, nationality, species membership. Cambridge (Mass.): Harvard University Press.

Nussbaum, M. (2010). Not For Profit. Why Democracy Needs the Humanities. Princeton: Princeton University Press. Traduction française de Chavel S. (2011). Les émotions démocratiques, comment former le citoyen $\mathrm{duXXI} \mathrm{I}^{\mathrm{eme}}$ siècle ? Paris: Flammarion.

Nussbaum , M. (2011). Creating Capabilities: The Human Development Approach. Cambridge. MA: Harvard University Press.

Ozouf, M (1992). Article « Fraternité ». In F. Furet et M. Ozouf (Dir.), Dictionnaire critique de la Révolution française. Paris : Flammarion.

Putnam, H. (2011 [1976]). Literature, Science and Reflection, New Literary History, vol. 7, n 3 , 488-89. 
Revault d'Allonnes, M. (2008). L'homme compassionnel. Paris : Seuil.

Zimbardo, P. (2007). The Lucifer Effect. How Good People Turn Evil. London: Rider.

\section{NOTES}

1. Extraits de la synthèse de la consultation nationale sur les programmes français d'Enseignement Moral et Civique de 2015 : «La plupart des contributions font état de réserves spécifiques émises par les équipes concernant l'évaluation des compétences relatives à la culture de la sensibilité, aux sentiments, aux émotions, au jugement moral : « Les enseignants expriment leur réticence face à l'évaluation de la culture de la sensibilité qui viserait la personnalité de l'élève et constituerait une forme d'intrusion dans sa vie personnelle ».[...] Dans une autre, ceux du second degré notent : " Des enseignants s'interrogent sur l'évaluation de certaines compétences qu'ils qualifient "d'abstraites" ou de subjectives, par exemple "être capable de prendre soin de soi", "être capable d'empathie", "se sentir membre d'une collectivité ». Texte accessible sur le site de l'APHG : https://www.aphg.fr/ (consulté le 1/11/2019).

2. Sur les différentes approches philosophiques possibles de la notion, voir: Chalier, C. (2003) ; Mattéi, B., "Penser la fraternité », intervention du 10 novembre 2004 dans le cadre du groupe d'études «La philosophie au sens large» animé par Pierre Macherey, https:// philolarge.hypotheses.org/files/2017/09/10-11-2004_Matt\%C3\%A9i.pdf, consulté le 2/02/2020.

3. Voir les analyses rousseauistes de la pitié dans l'Émile ou le Second discours sur l'origine et les fondements de l'inégalité parmi les hommes.

4. La décision du 6 juillet 2018 du Conseil Constitutionnel reconnaît pour la première fois la fraternité comme un principe à valeur constitutionnelle.

5. On peut considérer, selon l'usage, que la différence entre émotion et sentiment tient à leur temporalité différente. L'émotion est généralement assez labile, momentanée, tandis que le sentiment est plus ancré, plus durable. L'émotion est aussi perçue comme incarnée là où le sentiment a une dimension plus spirituelle.

6. Aristote, Rhétorique, $1385 \mathrm{~b} 13 \mathrm{sqq}$.

7. Descartes envisage la "générosité " en un sens très large comme une passion qui conduit à s'estimer à sa juste valeur et qui peut faire contrepoids à d'autres passions destructrices plus puissantes que la raison. On peut par exemple faire jouer la passion de la générosité qui pousse à défendre son honneur contre la passion de la peur qui pousse à la lâcheté. Voir Descartes, R. (1996). Les passions de l'âme, Paris : Vrin, Euvres complètes, Tome XI, art. 211, p. 487.

8. Clark, C. (1997); Hoffman, M. (2000).

9. Voir l'ouvrage dirigé par Bronislaw Baczko: Baczko, B. (éd). (1982). Une éducation pour la démocratie, textes et projets de l'époque révolutionnaire, Paris : Garnier frères.

10. Le site du CRDP de Reims restitue les différentes prises de position publiques sur le projet de parrainage d'enfants déportés par des élèves de $\mathrm{CM} 2$, notamment celles des associations professionnelles d'enseignants, http://www.cndp.fr/crdp-reims/memoire/enseigner/ memoire_vichy/14shoah_cm2.htm\#injonction, consulté le 2/03/2020.

11. Elle rattache cette capacité à la capabilité 7 qui porte sur l'affiliation : « être capable de vivre avec et pour les autres, de reconnaître et d'être attentif à d'autres êtres humains, de prendre part à différents types d'interactions sociales, d'être capable d'imaginer la situation d'autrui » (Nussbaum, 2011/2012: 56).

12. Les programmes français d'Enseignement Moral et Civique de 2015 s'efforcent de penser et de promouvoir cette articulation entre sensibilité et raison, mais les modifications publiées au Journal Officiel du 21/07/2018, tout en conservant la double référence à la «culture de la 
sensibilité » et à la "culture du jugement", ne détaillent plus de façon aussi explicite les démarches propices à cet apprentissage.

13. « Si je lis Le voyage au bout de la nuit de Céline, je n'apprends pas que l'amour n'existe pas, que tous les êtres humains sont odieux et haineux (même si -et je suis sûr que ce n'est pas le cas- ces propositions devaient être vraies). Ce que j'apprends est à voir le monde comme il a l'air d'être pour quelqu'un qui est sûr que cette hypothèse est correcte. » Putnam, H. (2011 [1976], p. 54).

\section{ABSTRACTS}

Martha Nussbaum, a contemporary American philosopher, has developed a stimulating reflection on the role of emotions in the education of citizens (Nussbaum, 2010/2011). This article proposes to demonstrate that her analyzes of the nature and effects of rational compassion shed new light on fraternity education in school. She develops pedagogical paths articulating the sensitive experience of the pupils and the rational discussion of the values involved in various situations concerning political and moral choices. She refers to the experiences of great education thinkers such as Tagore to encourage educators to tap into the valuable resources of literature and play.

Martha Nussbaum, philosophe américaine contemporaine, a développé une réflexion stimulante sur la place des émotions dans l'éducation des citoyens (Nussbaum, 2010/2011). Cet article propose de montrer dans quelle mesure ses analyses sur la nature et les effets de la compassion rationnelle permettent d'éclairer d'un jour nouveau l'éducation à la fraternité à l'école. Elle propose des pistes pédagogiques articulant l'expérience sensible des élèves et la discussion rationnelle des valeurs en jeu dans diverses situations engageant des choix politiques et moraux. Elle s'appuie sur les expériences de grands penseurs de l'éducation comme Tagore pour encourager les éducateurs à puiser dans les ressources précieuses que constituent la littérature et le jeu.

\section{INDEX}

Mots-clés: compassion, formation du citoyen, littérature, jeu

Keywords: compassion, citizen education, literature, play

\section{AUTHOR}

\section{STÉPHANIE PÉRAUD-PUIGSÉGUR}

INSPE d'Aquitaine, Université de Bordeaux, Laboratoire SPH, Sciences, Philosophie, Humanités (EA 4574) 